Original Article

\title{
SYNTHESIS, CHARACTERIZATION, BIOLOGICAL EVALUATION AND DOCKING OF SOME NOVEL SUBSTITUTED 1, 3-THIAZINE DERIVATIVES
}

\author{
RAVINDAR BAIRAM1 ${ }^{*}$, SRINIVASA MURTHY MUPPAVARAPU² AND SIVAN SREEKANTH ${ }^{3}$
}

${ }^{1}$ Department of Pharmaceutical Chemistry, Center for Pharmaceutical Sciences, New IST, Jawaharlal Nehru Technological University, Kukatpally, Hyderabad, Telangana 500085, India, ${ }^{2}$ Department of Pharmaceutical Chemistry, Vignan Institute of Pharmaceutical Sciences, Hyderabad, Telangana 508284, India, ${ }^{3}$ Department of Chemistry, Nizam College, Osmania University, Hyderabad, Telangana 500001, India Email: ravindarpharma@gmail.com

Received: 30 Nov 2016 Revised and Accepted: 30 Jan 2017

\section{ABSTRACT}

Objective: Chalcones and their heterocyclic analogs represent an important class of small molecules which have a wide range of pharmacological activities. Therefore, in this study, synthesis and anticonvulsant and antimicrobial activities of some new 1, 3-thiazines have been extensively discussed.

Methods: The reaction mixture of 4-tert-butylcyclohexanone on Claisen-Schmidt condensation with various aromatic aldehydes in the presence of dilute sodium hydroxide afforded the corresponding chalcones. Further, these compounds were subjected to cocondensation with thiourea, in the presence of isopropanol, catalyzed by aqueous potassium hydroxide to form 4-aryl 8-arylidene-5, 6-dihydro-2-imino-6-methyl-4H, 7H-(1, 3) benzothiazines. The structures of the newly synthesized compounds have been established on the basis of their spectral analysis. The newly synthesized compounds have been tested for their biological screening. Antimicrobial activity by cup plate agar diffusion method and antiepileptic activity by pentylenetetrazole (PTZ) induced seizures model, using diphenyl hydantain as standard, and also they are subjected to molecular properties prediction, toxicity, drug-likeness, lipophilicity and solubility parameters determination were done by using Osiris program, Molsoft, Prototox and ALOGPS 2.1 software. The binding mode of the synthesized compounds with active protein site has been predicted using docking method.

Results: Most of the compounds have shown good anticonvulsant as well as antimicrobial activities, but it is less than the standard drugs. 1, 3thiazines derivatives were more potent, and among them, compounds $\mathrm{TB}_{5}$ and $\mathrm{TB}_{7}$ were the most active compounds in these series; $\mathrm{TB}_{5}$ which contains isopropyl phenyl moiety, was shown moderate potent activity with onset of convulsion at 14.1 min and $\mathrm{TB}_{7}$ containing 3, 4, 5trimethoxyphenyl substituents on the thiazine moiety was more potent as it has prolonged the onset of convulsions by 18.7 min. Whereas in the case of antimicrobial activity of the compounds, from the results we have observed that $\mathrm{TB}_{5}$ have been shown greatest antimicrobial activity in all the bacterial and fungal strains, $\mathrm{TB}_{2}$ also shown superior activity, the others have been shown good antimicrobial activity.

Conclusion: According to the activity studies, it is observed that the synthesis and antimicrobial as well as anticonvulsant activities of novel 1, 3thiazine derivatives have been shown better activity. Moreover molecular docking results give an insight into how further modification of the lead compound can be carried out for higher inhibitory activity. In particular, compounds with electron withdrawing substituents along with lipophilic methoxyl and isopropyl groups were more potent.

Keywords: Thiazine, Anticonvulsant, Antimicrobial activities, Molecular docking

(C) 2016 The Authors. Published by Innovare Academic Sciences Pvt Ltd. This is an open access article under the CC BY license (http://creativecommons.org/licenses/by/4.0/) DOI: http://dx.doi.org/10.22159/ijpps.2017v9i3.16406

\section{INTRODUCTION}

Research in pharmaceutical chemistry renders a vital role in the discovery of newer therapeutic agents. The heterocyclic compounds [1] which contain nitrogen, sulphur and oxygen possess an enormous significance in the field of medicinal chemistry, heterocyclic compounds were abundant in nature and have acquired more importance, because their structural subunits were exhibited in many natural products such as vitamins, hormones, antibiotics, etc. 1, 3-thiazines which contain nitrogen and sulfur in their sixmember heterocyclic ring system (N-C-S), as a pharmacophore and it was found to be fairly stable, also in some other medicinally important compounds like xylazine (agonist at the $\alpha_{2}$-adrenergic receptor is used for sedation, anesthesia, muscle relaxation, and analgesia in animals) [2], and chlormezanone (used as an anxiolytic and a muscle relaxant) [3]. Thiazine derivative moiety results has shown diverse biological and pharmaceutical profiles such as anticonvulsant [4], antimicrobial [5-10], analgesic, antiinflammatory and ulcerogenic [11-13], anticancer [14-18], antidiabetic [19], immunotropic [20], antianxiety [21], insecticidal and pesticidal [22], antitubercular [23], anthelmintic [24], anesthetic [25], antiviral [26] activities etc. The treatment for bacterial infection currently represents a significant therapeutic challenge throughout the world. Since this trend has been changing, there is a due need for the development of new antibacterial agents with divergent and unique structural features, which operate via different mechanisms of actions to those of existing antimicrobial agents. Moreover, thiazine nucleus was a pharmacophore of cephalosporins that occupy a very important place in the field of antibiotics. Search for new molecules continues because of the fast development of microbial resistance towards existing molecules, and therefore new 1, 3-thiazine derivatives have been synthesized with an objective to get potent antimicrobial agents, in the present work the reaction involves the synthesis of chalcones by using various aryl aldehydes and aryl ketone (Claisen-Schmidt condensation reaction). Different chalcone derivatives were used as the initial material for the synthesis of new thiazines.

Though a number of drugs are available in the market, they are not up to the mark. The ALOGPS method is a part of the ALOGPS 2.1 programmer [27], which is used to predict lipophilicity and aqueous solubility of compounds. The lipophilicity calculation within this program is based on the associative neural network approach and the efficient partition algorithm. The LogKow (Kow-WIN) program estimates the log octanol/water partition coefficient (log p) of organic chemicals and drugs. An insufficient aqueous solubility is likely to impede bioavailability of the drugs. It is well known that insufficient solubility of drugs can lead to poor absorption. 


\section{MATERIALS AND METHODS}

\section{General}

All the chemicals and solvents are procured from Sigma-Aldrich and Merck, India. The melting points of the synthesized compounds have been determined in open capillary tubes and are uncorrected. The infra-red (IR) spectra of the compounds have been recorded on Perkin Elmer Fourier Transform infrared (FTIR) spectrometer (Model Shimadzu 8700) in $\mathrm{KBr}$ discs method. ${ }^{1} \mathrm{H}$ and ${ }^{13} \mathrm{C}$ nuclear magnetic resonance (NMR) spectra have been recorded on Bruker $400 \mathrm{MHZ}$ AVANCE instrument in deuterated chloroform $\left(\mathrm{CDCl}_{3}\right)$ at $399 \mathrm{MHz}$. The chemical shifts $(\delta)$ have been reported in parts per million (ppm) downfield from internal reference Tetramethylsilane (TMS). Mass spectra have been recorded on Mass spectrophotometer (Model Shimadzu 2010A) by LC-MS, using dimethyl sulfoxide as a solvent. All the compounds have been shown satisfactory chemical analysis, the homogencity of the compounds have been checked by thin layer chromatography (TLC) using silica gel glass plates containing 60 F-254 and n-hexane and ethyl acetate $(8: 2)$ as mobile phase, and visualization on TLC was achieved by using ultraviolet light. All the compounds have been purified by column chromatography (Merck), pentylenetetrazole (PTZ) was procured from sigma and it was dissolved in normal saline. The healthy albino mice (Wistar, 100-150 g, 5-6 w) were obtained from Mahaveer enterprises, Kolkata.

\section{Experimental part chemical synthesis}

\section{General procedure}

\section{Preparation of 2, 6-dibenzylidene-4-tert-butyl cyclohexanone}

The substituted aromatic aldehyde $(0.02 \mathrm{~mol})$ has been added to the reaction mixture of $30 \mathrm{ml}$ of $10 \%$ sodium hydroxide and 4-tertbutylcyclohexanone $(0.01 \mathrm{~mol})$ in $50 \mathrm{ml}$ of ethyl alcohol, which is maintained at $20-25^{\circ} \mathrm{C}$ (fig. 1). This reaction mixture is magnetically stirred at $500 \mathrm{rpm}$ for a period of $2 \mathrm{hr}$. The completion of reaction has been confirmed by TLC method of estimation. Subsequently, after the $2 \mathrm{hr}$ the reaction mixture was stored in a refrigerator at $0-4$ ${ }^{\circ} \mathrm{C}$ temperature for a period of $8 \mathrm{hr}$. Finally, the obtained residue product was filtered, and washed with ice cold water followed by ice-cold ethanol than the washed product was dried and recrystallized from dimethylformamide gave as yellow crystals [27, 28]. Yields were from $65 \%$ to $95 \%$, mp. 126.6-127.0 UV ( $\lambda$ max) of 3a: $396 \mathrm{~nm}$; ${ }^{1} \mathrm{H}$ NMR of $1 \mathrm{a}:\left(\mathrm{CDCl}_{3}-\mathrm{d}_{6}\right): \delta 0.75\left(\mathrm{~m}, 2 \times \mathrm{CH}_{3}, 6 \mathrm{H}\right), 0.78$ $\left(\mathrm{d}, \mathrm{CH}_{3}, 3 \mathrm{H}\right), 1.32-1.34(\mathrm{~d}, \mathrm{CH}, 1 \mathrm{H}), 1.83-1.87\left(\mathrm{~m}, \mathrm{CH}_{2} 2 \mathrm{H}\right), 2.88\left(\mathrm{~s}, \mathrm{CH}_{2}\right.$, $2 \mathrm{H})$, 7.28-7.41 (m, ArH, 10H), 7.55 (s, methine $\mathrm{H}, 2 \mathrm{H})$. IR (KBr) of 3c: $1657 \mathrm{~cm}^{-1}$ (C=0) 1598, 1554, 1506, $1462 \mathrm{~cm}^{-1}$ (aromatic), $834 \mathrm{~cm}^{-1}$ $(\mathrm{C}=\mathrm{C})$; Mass of 3a: MS (ESI): $m / \mathbf{z}=388.9$ [M $\left.\mathrm{M}^{1}+1\right]$.

Preparation of 8-benzylidene-6-tert-butyl-4-phenyl-5, 6, 7, 8tetrahydro-1H-benzo-1, 3-thiazin-2-imine

To a mixture containing 2, 6-dibenzylidene-4-tert-butylcyclohexanone $(0.01 \mathrm{~mol})$, thiourea $(0.015 \mathrm{~mol})$ and potassium hydroxide $(0.01 \mathrm{~mol})$ were dissolved in specified amount $(5 \mathrm{ml})$ of water which was refluxed in isopropyl alcohol $(100 \mathrm{ml})$ for a period of $16 \mathrm{hr}$. Later, the formation of the product was confirmed by thin layer chromatography (TLC). Finally the left over solvent was removed under reduced pressure by distillation and the residue obtained was cooled, treated with ice cold water, followed by ice cold ethanol, then filtered, dried and the dried residue was purified by silica gel column chromatography (Ethyl acetate/Hexane) to give respective compounds $\left(\mathrm{TB}_{1}-\mathrm{TB}_{12}\right)$ and the precipitate obtained was recrystallized from ethyl alcohol $[29,30]$. The physical data of the compounds is given in table 1 .

\section{Spectral data of the synthesized compounds}

\section{8-benzylidene-6-tert-butyl-4-phenyl-5,6,7,8-tetrahydro-1H- benzo[d][1,3] thiazin-2 $(4 \mathrm{H})$-imine $\left(\mathrm{TB}_{1}\right)$}

Yellow solid, Yield $90 \%$; mp $184-186{ }^{\circ} \mathrm{C} . \mathrm{R}_{\mathrm{f:}}$ 0.7; IR $\left(\mathrm{KBr}, \mathrm{cm}^{-1}\right)$ : 3399.9 (Imine NH-), 3207.0 (cyclic NH-), 1609.3 (C=N), 1077 (C-N), 1476.2 $\left(\mathrm{CH}_{3}-\right)$. ${ }^{1} \mathrm{H}$ NMR $\left(400 \mathrm{MHz}, \mathrm{CDCl}_{3}, \delta \mathrm{ppm}\right): 0.75\left(\mathrm{~m}, 2 \times \mathrm{CH}_{3}\right.$, $6 \mathrm{H}), 0.78\left(\mathrm{~d}, \mathrm{CH}_{3}, 3 \mathrm{H}\right), 1.32-1.34(\mathrm{~d}, \mathrm{CH}, 1 \mathrm{H}), 1.83-1.87\left(\mathrm{~m},-\mathrm{CH}_{2-}, 2 \mathrm{H}\right)$, $2.88\left(\mathrm{~s}, \mathrm{CH}_{2}, 2 \mathrm{H}\right), 4.93(\mathrm{~s}, \mathrm{CH}-\mathrm{S}, 1 \mathrm{H}), 6.51(\mathrm{~s}$, imine $\mathrm{NH}, 1 \mathrm{H}), 6.64(\mathrm{~s}$, cyclic NH, 1H), 7.25-7.27 (d, Ar-H, 1H), 7.28-7.41(m, Ar-H, 9H), 7.55 (s, methine $\mathrm{H}, 1 \mathrm{H}$ ). [13]C NMR (400 MHz, DMSO-d 6 , $\delta$ ppm): 178.47, 152.03, 151.79. 151.68, 148.41, 142.58, 139.67, 139.26, 137.58, 135.88, 134.53, 133.84, 132.36, 127.53 (C-2' and C-6'), 127.41, (C-3" and $\left.\mathrm{C}^{-} 5^{\prime \prime}\right), 125.82,125.42,118.87,44.15$, (-CH2-, C of isobutyl group at C-4"); 43.95, 36.1, 35.9; 31.54 (-CH-, C of isobutyl group at C-4"). MS (ESI): $\mathrm{m} / \mathrm{z}=388.9$ [Mำ1].

8-(3,4-dimethoxybenzylidene)-6-tert-butyl-4-(3,4-dimethoxyphenyl)-5,6,7,8-tetrahydro-1H-benzo[d][1,3]thiazin-2(4H)imine $\left(\mathrm{TB}_{2}\right)$

Yellowish solid, Yield 78\%; mp 166-168 ${ }^{\circ} \mathrm{C}$. $\mathrm{R}_{\mathrm{f}}$ 0.7; IR $\left(\mathrm{KBr}, \mathrm{cm}^{-1}\right)$ : 3426.0 (Imine NH-), 3098.0 (cyclic NH-), 1655.0 (C=N), 1067.0 (C$\mathrm{N}), 1250.0(\mathrm{C}-\mathrm{O}-\mathrm{C}), 1458.0\left(\mathrm{CH}_{3}-\right) .{ }^{1} \mathrm{H}$ NMR $\left(400 \mathrm{MHz}, \mathrm{CDCl}_{3}, \delta \mathrm{ppm}\right)$ : 0.67-0.86 $\left(\mathrm{m}, 3 \times \mathrm{CH}_{3}, 9 \mathrm{H}\right), 1.20-1.23(\mathrm{~d}, \mathrm{CH}, 1 \mathrm{H}), 1.82-1.88\left(\mathrm{~m}, \mathrm{CH}_{2}\right.$, $2 \mathrm{H}), 1.93-2.07\left(\mathrm{~m}, \mathrm{CH}_{2} 2 \mathrm{H}\right), 3.41-3.88\left(\mathrm{~m}\left(4 \times \mathrm{OCH}_{3}, 12 \mathrm{H}\right), 18 \mathrm{H}\right), 4.72$ (s, CH-S, 1H), 6.72 (s, imine NH, 1H), 6.74 (s, cyclic NH, 1H), 6.74$6.98(\mathrm{~m}, \mathrm{ArH}, 6 \mathrm{H}), 8.93$ (s, methine, 1H). [13]C NMR ((400 MHz, DMSO-d $_{6}, \delta$ ppm): $174.47,148.47,147.25,140.413,135.05,129.58$, $128.41,127.44,126.58,122.88,114.66,58.42,43.62,40.61,39.98$, 27.49. MS (ESI): $\mathrm{m} / \mathrm{z}=509.1\left[\mathrm{M}^{1}+1\right]$.

4-((6-tert-butyl-4-(4-(dimethylamino)phenyl)-2-imino-1,2,6,7tetrahydro-4H-benzo[d][1,3]thiazin-8(5H)-ylidene)methyl)- $\mathrm{N}$, $\mathrm{N}$-dimethylbenzenamine ( $\mathrm{TB}_{3}$ ) (fig. 2)

Light brown solid, Yield 69\%; mp 142-144 ${ }^{\circ} \mathrm{C}$; $\mathrm{R}_{\mathrm{f}}$ 0.7; IR $\left(\mathrm{KBr}, \mathrm{cm}^{-1}\right)$ : 3425.0 (Imine NH-), 3069.0 (cyclic NH-), 1654.0(C=N), 1116.0 (C-N), 1454.0 $\left(\mathrm{CH}_{3}-\right) .{ }^{1} \mathrm{H}$ NMR $\left(400 \mathrm{MHz}, \mathrm{CDCl}_{3}, \delta \mathrm{ppm}\right): 0.67-0.74\left(\mathrm{~m}^{-\mathrm{CH}_{3}}\right.$, $3 \mathrm{H}), 0.81-2.23\left(\mathrm{~m}, 2 \times \mathrm{CH}_{3}, 6 \mathrm{H}\right), 1.25(\mathrm{~d}, \mathrm{CH}, 1 \mathrm{H}), 2.79-2.82\left(\mathrm{~m}, 2 \times \mathrm{CH}_{2}\right.$ $4 \mathrm{H}), 2.84-3.07\left(\mathrm{~m}, 4 \times \mathrm{NCH}_{3}, 12 \mathrm{H}\right), 5.38(\mathrm{~s}, \mathrm{CH}-\mathrm{S}, 1 \mathrm{H}), 6.55$ (s, imine $\mathrm{NH}, 1 \mathrm{H}), 6.66(\mathrm{~d}$, cyclic NH, 1H), 6.67-7.65 (m, ArH, 8H), 7.67 (s, methine $\mathrm{H}, 1 \mathrm{H})$. MS (ESI): $\mathrm{m} / \mathrm{z}=473.2\left[\mathrm{M}^{1}+1\right]$.

8-(4-methoxybenzylidene)-6-tert-butyl-4-(4-methoxyphenyl)5,6,7,8-tetrahydro-1H-benzo[d][1,3] thiazin-2 $(4 \mathrm{H})$-imine $\left(\mathrm{TB}_{4}\right)$

Yellowish solid, Yield 89\%; mp 189-192 ${ }^{\circ} \mathrm{C}$; $\mathrm{R}_{\mathrm{f}} 0.4$; IR $\left(\mathrm{KBr}, \mathrm{cm}^{-1}\right): 3384.0$ (Imine NH-), 3201.0 (cyclic NH-), 1601.1 (C=N), 1031.0 (C-N), 1248(C-OC), $1476.0\left(\mathrm{CH}_{3}{ }^{-}\right) .{ }^{1} \mathrm{H}$ NMR $(400 \mathrm{MHz}, \mathrm{CDCl} 3, \delta \mathrm{ppm}): 0.76-0.79(\mathrm{~m}, 3 \times$ $\left.\mathrm{CH}_{3}, 9 \mathrm{H}\right), 0.84-0.92\left(\mathrm{~m}, \mathrm{CH}_{2}, 2 \mathrm{H}\right), 1.28-1.34\left(\mathrm{~m}, \mathrm{CH}_{2}, 1 \mathrm{H}\right), 1.81-1.89(\mathrm{~m}$, $\left.\mathrm{CH}_{2} 1 \mathrm{H}\right), 1.96-2.02(\mathrm{~m}, \mathrm{CH}, 1 \mathrm{H}), 3.81-3.83\left(\left(\mathrm{~m}, 2 \times \mathrm{OCH}_{3}, 6 \mathrm{H}\right), 6.44\right.$ (s, CH-S, $1 \mathrm{H}), 6.61$ (s, imine NH, 1H), 6.88 (s, cyclic NH, 1H), 6.88-6.92 (m, ArH, $4 \mathrm{H}), 7.19-7.23(\mathrm{~m}, \mathrm{ArH}, 4 \mathrm{H}), 7.57$ (s, methine $\mathrm{H}, 1 \mathrm{H})$. [13]C NMR ((400 MHz, DMSO-d $6, \delta$ ppm):174.37, 149.14, 148.90, 135.27, 135.27, 128.50, $122.75,119.33,114.36,111.98,58.33,55.78,43.73,39.55,27.87$; MS (ESI): $\mathrm{m} / \mathrm{z}=448.9\left[\mathrm{M}^{1}+1\right]$.

8-(4-isopropylbenzylidene)-6-tert-butyl-4-(4-isopropylphenyl)-5,6,7,8-tetrahydro-1H-benzo[d][1,3]thiazin-2(4H)imine ( $\left.\mathrm{TB}_{5}\right)$

White solid, Yield 88\%; $\mathrm{mp} 180-182^{\circ} \mathrm{C}$; $\mathrm{R}_{\mathrm{f}}$ 0.5; IR ( $\left.\mathrm{KBr}, \mathrm{cm}^{-1}\right): 3426.0$ (Imine NH-), 3064.0 (cyclic NH-), $1650.0(\mathrm{C}=\mathrm{N}), 1159.0(\mathrm{C}-\mathrm{N})$, $1457.0\left(\mathrm{CH}_{3}-\right)$. ${ }^{1} \mathrm{H}$ NMR $\left(400 \mathrm{MHz}, \mathrm{CDCl}_{3}, \delta \mathrm{ppm}\right): 0.71-0.86(\mathrm{~m}, 3 \times$ $\left.\mathrm{CH}_{3}, 9 \mathrm{H}\right), 0.87-0.97\left(\mathrm{~m}, \mathrm{CH}_{2}, 2 \mathrm{H}\right), 1.28-1.34\left(\mathrm{~m}, \mathrm{CH}_{2}, 1 \mathrm{H}\right), 1.81-1.89$ $\left(\mathrm{m}, \mathrm{CH}_{2} 1 \mathrm{H}\right), 1.96-2.40(\mathrm{~m}, \mathrm{CH}, 1 \mathrm{H}), 2.40-2.44(\mathrm{~m}, \mathrm{CH}, 1 \mathrm{H}), 3.13-$ $3.89\left(\left(\mathrm{~m}, 2 \times \mathrm{CH}_{3}, 6 \mathrm{H}\right), 4.84(\mathrm{~s}, \mathrm{CH}-\mathrm{S}, 1 \mathrm{H}), 6.4(\mathrm{~s}\right.$, imine $\mathrm{NH}, 1 \mathrm{H}), 6.88$ (s, cyclic $\mathrm{NH}, 1 \mathrm{H}), 6.88-6.92(\mathrm{~m}, \mathrm{ArH}, 4 \mathrm{H}), 7.61-7.73(\mathrm{~m}, \mathrm{ArH}, 4 \mathrm{H})$, 7.75 (s, methine $\mathrm{H}, 1 \mathrm{H})$. MS (ESI): $\mathrm{m} / \mathrm{z}=742.7\left[\mathrm{M}^{1}+1\right]$.

8-(4-chlorobenzylidene)-6-tert-butyl-4-(4-chlorophenyl)-5,6, 7,8-tetrahydro-1H-benzo[d] $[1,3]$ thiazin-2 $(4 \mathrm{H})$-imine $\left(\mathrm{TB}_{6}\right)$

White solid, Yield $80 \%$; $m p$ 222-224 ${ }^{\circ} \mathrm{C}$; $\mathrm{R}_{\mathrm{f}}$ 0.7; IR $\left(\mathrm{KBr}, \mathrm{cm}^{-1}\right)$ : 3424.0 (Imine NH-), 3275.5 (cyclic NH-), 1615.0 (C=N), 1089.0 (C$\mathrm{N}), 1487.0\left(\mathrm{CH}_{3}-\right)$; Ar-Cl-809.0. ${ }^{1} \mathrm{H}$ NMR $\left(400 \mathrm{MHz}, \mathrm{CDCl}_{3}, \delta \mathrm{ppm}\right)$ : $0.75\left(\mathrm{~m}, 2 \times \mathrm{CH}_{3}, 6 \mathrm{H}\right), 0.78\left(\mathrm{~d}, \mathrm{CH}_{3}, 3 \mathrm{H}\right), 1.32-1.34(\mathrm{~d}, \mathrm{CH}, 1 \mathrm{H}), 1.83-$ $1.87\left(\mathrm{~m},-\mathrm{CH}_{2}-, 2 \mathrm{H}\right), 2.88\left(\mathrm{~s}, \mathrm{CH}_{2}, 2 \mathrm{H}\right), 4.93(\mathrm{~s}, \mathrm{CH}-\mathrm{S}, 1 \mathrm{H}), 6.51(\mathrm{~s}$, imine $\mathrm{NH}, 1 \mathrm{H}), 6.64$ (s, cyclic NH, 1H), 7.25-7.27 (d, Ar-H, 1H), 7.28-7.41(m, Ar-H, 9H), 7.55 (s, methine H, 1H). MS (ESI): $\mathrm{m} / \mathrm{z}=456.7\left[\mathrm{M}^{1}+1\right]$.

8-(4-fluorobenzylidene)-6-tert-butyl-4-(4-fluorophenyl)-5,6, 7,8-tetrahydro-1H-benzo[d] $[1,3]$ thiazin-2 $(4 \mathrm{H})$-imine $\left(\mathrm{TB}_{7}\right)$

White solid, Yield 82\%; mp 243-245 ${ }^{\circ} \mathrm{C}$; $\mathrm{R}_{\text {f: }} 0.6$; IR $\left(\mathrm{KBr}, \mathrm{cm}^{-1}\right)$ : 3256.0 (Imine NH-), 2921.0 (cyclic NH-), 1693.0 (C=N), 1115.0 (C- 


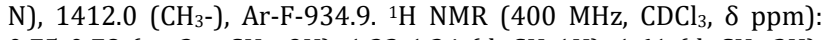
$0.75-0.78\left(\mathrm{~m}, 3 \times \mathrm{CH}_{3}, 9 \mathrm{H}\right), 1.32-1.34(\mathrm{~d}, \mathrm{CH}, 1 \mathrm{H}), 1.61\left(\mathrm{~d}, \mathrm{CH}_{2} 2 \mathrm{H}\right)$, 1.83-2.03 (m, $\left.\mathrm{CH}_{2}, 2 \mathrm{H}\right), 4.93(\mathrm{~s}, \mathrm{CH}-\mathrm{S}, 1 \mathrm{H}), 6.51(\mathrm{~s}$, imine $\mathrm{NH}, 1 \mathrm{H})$, 6.64 (s, cyclic NH, 1H), 7.25-7.39 (m, ArH, 8H), 7.59 (s, methine H, 1H). [13]C NMR ((400 MHz, DMSO-d $6, \delta$ ppm): 190.28 (C-1), 184.30, $172.64,163.28,154.62,150.37,131.99,128.19,127.95,112.70$, 107.50, 59.33, 44.33, 40.57, 39.94, 27.88. MS (ESI): $\mathrm{m} / \mathrm{z}=425.1$ $\left[\mathrm{M}^{1}+1\right]$.

8-(3,4,5-trimethoxybenzylidene)-6-tert-butyl-4-(3,4,5-trimethoxyphenyl)-5,6,7,8-tetrahydro-1H-benzo[d][1,3]thiazin2(4H)-imine $\left(\mathrm{TB}_{8}\right)$

Yellowish solid, Yield 94\%; mp 210-212 ${ }^{\circ} \mathrm{C}$; Rf: 0.5 ; IR (KBr, $\mathrm{cm}^{-1}$ ): 3663.0 (Imine $\mathrm{NH}-$ ), 3291.0 (cyclic $\mathrm{NH}-$ ), $1673.0(\mathrm{C}=\mathrm{N})$, 1065.0 (C-N), 1263.0(C-O-C), Ar-Trisubstituted, $1419.0\left(\mathrm{CH}_{3}-\right)$. ${ }^{1} \mathrm{H}$ NMR $\left.\left(400 \mathrm{MHz} \mathrm{CDCl}_{3}, \delta \mathrm{ppm}\right): 0.71-0.79\left(\mathrm{~m}, \mathrm{CH}_{3}\right)_{2}, 6 \mathrm{H}\right), 0.81-$ $0.83\left(\mathrm{~d}, \mathrm{CH}_{3}, 3 \mathrm{H}\right), 1.22(\mathrm{~s}, \mathrm{CH}, 1 \mathrm{H}), 1.24-1.99\left(\mathrm{~m}, \mathrm{CH}_{2} 2 \mathrm{H}\right), 2.08-$ $2.99\left(\mathrm{~s}, \mathrm{CH}_{2}, 1 \mathrm{H}\right), 2.48\left(\mathrm{~s}, \mathrm{CH}_{2}, 1 \mathrm{H}\right), 3.61-3.84\left(\mathrm{~m}\left(6 \times \mathrm{OCH}_{3}, 18 \mathrm{H}\right)\right.$, $4.44(\mathrm{~s}, \mathrm{CH}-\mathrm{S}, 1 \mathrm{H}), 6.57$ (s, imine NH, 1H), 6.58 (s, cyclic NH, 1H), 6.62-6.64 (m, ArH, 4H), $8.96(\mathrm{~s}$, methine $\mathrm{H}, 1 \mathrm{H}) . \mathrm{MS}(\mathrm{ESI}): \mathrm{m} / \mathrm{z}=$ $569.1\left[\mathrm{M}^{1}+1\right]$.

4-((4-(4-aminophenyl)-6-tert-butyl-2-imino-1,2,6,7-tetrahydro4H-benzo[d][1,3]thiazin-8(5H)-ylidene)methyl) benzenamine (TB9)

Light brown solid, Yield 70\%; mp 156-158 ${ }^{\circ} \mathrm{C}$; $\mathrm{R}_{\mathrm{f}:} 0.6$; $\mathrm{IR}\left(\mathrm{KBr}, \mathrm{cm}^{-1}\right)$ : 3441.0 (Imine NH-), 3063.0 (cyclic NH-), 1653.0(C=N), $1092.0(\mathrm{C}-\mathrm{N})$, $1456.0\left(\mathrm{CH}_{3}-\right), 735.6\left(\mathrm{Ar}-\mathrm{NH}_{2}\right) .{ }^{1} \mathrm{H}$ NMR $\left(400 \mathrm{MHz}, \mathrm{CDCl}_{3}, \delta \mathrm{ppm}\right)$ : 0.67-0.75 (m, $\left.3 \times \mathrm{CH}_{3}, 9 \mathrm{H}\right), 1.13-1.19(\mathrm{~d}, \mathrm{CH}, 1 \mathrm{H}), 1.73-1.79\left(\mathrm{~m}, \mathrm{CH}_{2}\right.$, $2 \mathrm{H}), 1.95-2.05\left(\mathrm{~m}, \mathrm{CH}_{2}, 1 \mathrm{H}\right), 2.812 .85\left(\mathrm{~d}, \mathrm{CH}_{2}, 1 \mathrm{H}\right), 4.81-4.84(\mathrm{~d}, \mathrm{CH}-\mathrm{S}$, $1 \mathrm{H}), 6.96(\mathrm{~s}$, imine NH, 1H), 7.06 (s, cyclic NH, 1H),7.16-7.73 (m, ArH, $8 \mathrm{H}), 9.04(\mathrm{~s}$, methine $\mathrm{H}, 1 \mathrm{H}) .[13] \mathrm{C}$ NMR ((400 MHz, DMSO-d $6, \delta$ ppm): $174.48,163.36,162.52,160.94,139.22,133.88,131.51$, $129.56,128.31,122.14,116.08,114.83,57.89,43.69,39.35,27.96$. MS (ESI): $\mathrm{m} / \mathrm{z}=418.5\left[\mathrm{M}^{1}+1\right]$. 8-(4-ethylbenzylidene)-6-tert-butyl-4-(4-ethylphenyl)-5, 6, 7, 8tetrahydro-1H-benzo[d][1,3] thiazin-2(4H)-imine(TB 10$)$

Yellow solid, Yield $76 \%$; mp $178-180{ }^{\circ} \mathrm{C}$; $\mathrm{R}_{\mathrm{f}}$ 0.6; IR $\left(\mathrm{KBr}, \mathrm{cm}^{-1}\right)$ : 3380.0 (Imine $\mathrm{NH}-$ ), 3201.0 (cyclic $\mathrm{NH}-$ ), 2934.0 $\left(\mathrm{Ar}^{-} \mathrm{C}_{2} \mathrm{H}_{5}\right.$ ). 1611.0 $(\mathrm{C}=\mathrm{N}), 1060.0(\mathrm{C}-\mathrm{N}), 1475.0\left(\mathrm{CH}_{3}-\right), 735.6\left(\mathrm{Ar}^{\left.-\mathrm{NH}_{2}\right) .}{ }^{1} \mathrm{H}\right.$ NMR (400 $\left.\mathrm{MHz}, \mathrm{CDCl}_{3}, \delta \mathrm{ppm}\right): 0.76-0.79\left(\mathrm{~m}, 3 \times \mathrm{CH}_{3}, 9 \mathrm{H}\right), 0.82-0.86(\mathrm{~m}$, $\left.2 \times \mathrm{CH}_{2}, 4 \mathrm{H}\right), 0.92(\mathrm{~m}, \mathrm{CH}, 1 \mathrm{H}), 1.21-1.27\left(\mathrm{~m}, 2 \times \mathrm{CH}_{2}, 4 \mathrm{H}\right), 2.62-2.69$ $\left(\mathrm{m}, 2 \times \mathrm{CH}_{3}, 6 \mathrm{H}\right), 6.47(\mathrm{~s}, \mathrm{CH}-\mathrm{S}, 1 \mathrm{H}), 6.59(\mathrm{~s}$, imine NH, $1 \mathrm{H}), 7.20(\mathrm{~s}$, cyclic $\mathrm{NH}, 1 \mathrm{H}), 7.20-7.22(\mathrm{~m}, \mathrm{ArH}, 8 \mathrm{H}), 7.58$ (s, methine $\mathrm{H}, 1 \mathrm{H})$. [13]C NMR ((400 MHz, DMSO-d 6 , $\delta$ ppm): 174.48, 163.36, 162.52, 160.94, $139.22,133.88,131.51,129.56,128.31,122.14,116.08,114.83$, 57.89, 43.69, 39.35, 27.96. MS (ESI): $\mathrm{m} / \mathrm{z}=444.9\left[\mathrm{M}^{1}+1\right]$.

8-(3-Nitrobenzylidene)-6-tert-butyl-4-(3-nitrophenyl)-5,6,7,8tetrahydro-1H-benzo[d][1,3] thiazin-2 $(4 \mathrm{H})$-imine $\left(\mathrm{TB}_{11}\right)$

Light brown solid, Yield 65\%; mp 182-184 ${ }^{\circ} \mathrm{C}$; Rf: 0.7 ; $\mathrm{IR}\left(\mathrm{KBr}, \mathrm{cm}^{-1}\right)$ : 3418.0 (Imine NH-), 3087.0 (cyclic NH-), 1601.0(C=N), 1060.0(C-N), $1462.0\left(\mathrm{CH}_{3}-\right), 832.1\left(\mathrm{Ar}^{\left.-\mathrm{NO}_{2}\right) .}{ }^{1} \mathrm{H}\right.$ NMR $\left(400 \mathrm{MHz}, \mathrm{CDCl}_{3}, \delta \mathrm{ppm}\right)$ : 0.63-0.65 (d, $\left.\mathrm{CH}_{3}, 3 \mathrm{H}\right), 0.70-0.92\left(\mathrm{~m}, 2 \times \mathrm{CH}_{3}, 6 \mathrm{H}\right), 1.00-1.02(\mathrm{~d}, \mathrm{CH}$, $1 \mathrm{H}), 1.12-1.16\left(\mathrm{~m}, \mathrm{CH}_{2}, 2 \mathrm{H}\right), 1.71-2.37\left(\mathrm{~m}, \mathrm{CH}_{2}, 2 \mathrm{H}\right), 4.96-5.21(\mathrm{~m}, \mathrm{CH}-$ $\mathrm{S}, 1 \mathrm{H}), 6.42(\mathrm{t}$, imine $\mathrm{NH}, 1 \mathrm{H}), 6.57(\mathrm{~s}$, cyclic $\mathrm{NH}, 1 \mathrm{H}), 6.66-6.91(\mathrm{~d}$, ArH, 1H), 7.07-7.23 (d, ArH, 1H), 7.65-7.89(m, ArH, 4H), 7.92(d, ArH, 2H), 8.31 (s, methine $\mathrm{H}, 1 \mathrm{H}$ ). MS (ESI): $\mathrm{m} / \mathrm{z}=478.56$ [Mำ1].

4-((6-tert-butyl-4-(3,4-dihydroxyphenyl)-2-imino-1,2,6,7tetrahydro-4H-benzo[d][1,3] thiazin-8(5H)-ylidene)methyl) benzene-1,2-diol ( $\left.\mathrm{TB}_{12}\right)$

Light white solid, Yield $65 \%$; mp 177-179 ${ }^{\circ} \mathrm{C}$; $\mathrm{R}_{\mathrm{f}}$ 0.5; IR $\left(\mathrm{KBr}, \mathrm{cm}^{-1}\right)$ : 3444.0 (Imine NH-), 2951.0 (cyclic NH-), 1593.0(C=N), 1027.0(C-N), $1466.0\left(\mathrm{CH}_{3}-\right), 774.3$ (Di-substituted). ${ }^{1} \mathrm{H}$ NMR $\left(400 \mathrm{MHz}, \mathrm{CDCl}_{3}, \delta\right.$ ppm): 1.5-2.4 (m, $\left.\left(\mathrm{CH}_{2}\right)_{2}, 4 \mathrm{H}\right), 2.4\left(\mathrm{~m}, \mathrm{CH}_{2}, 2 \mathrm{H}\right), 4.3(\mathrm{~s}, \mathrm{CH}-\mathrm{S}, 1 \mathrm{H}), 6.9$ (s, imine, $1 \mathrm{H}), 6.9(\mathrm{~s}$, cyclic $\mathrm{NH}, 1 \mathrm{H}), 7.0-7.7(\mathrm{~m}, \mathrm{ArH}, 8 \mathrm{H}), 7.7(\mathrm{~s}$, methine, $1 \mathrm{H})$. [13]C NMR ((400 MHz, DMSO-d $6, \delta \mathrm{ppm}): 174.65$, $153.45,138.29,137.57,136.85,133.03,131.93,129.40,128.57$, 123.04, 114.60, 107.09, 104.71, 60.52, 58.60, 43.73, 40.60, 39.97, 27.40. MS (ESI): $\mathrm{m} / \mathrm{z}=451.4\left[\mathrm{M}^{1}+1\right]$.

Scheme of work<smiles>CC(C)(C)C1CC(C(C)(C)CCCCCC2CC(=O)CCC2C(C)(C)C)CC(=CBr)C1=O</smiles>

Fig. 1: a) Ar-CHO, substituted aromatic aldehydes, ethanol, sodium hydroxide (NaOH), b) thiourea, aqueous potassium hydroxide (KOH), isopropyl alcohol, reflux, $14 \mathrm{hr}$, reaction scheme for synthesis of title compounds

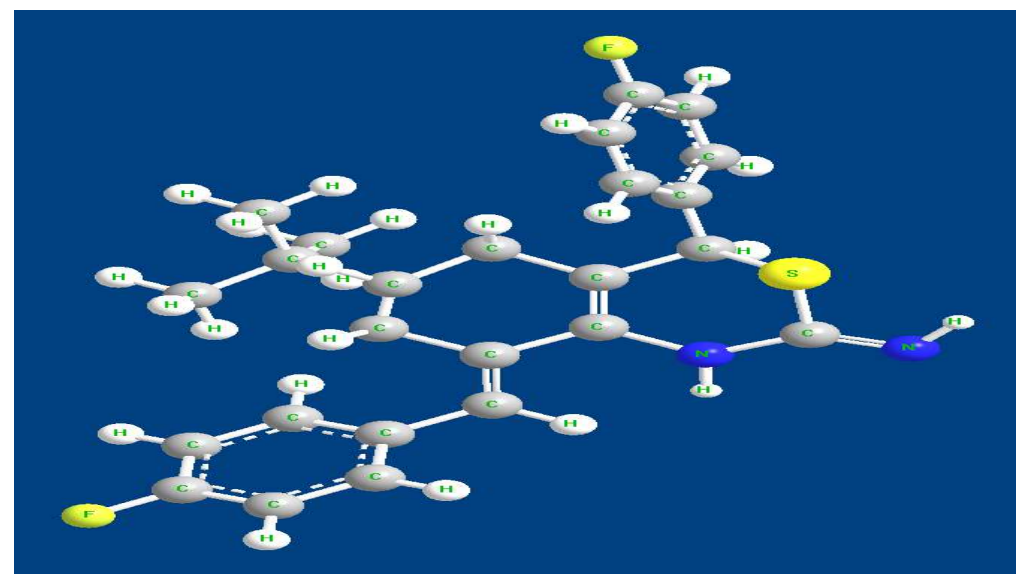

Fig. 2: 3 Dimensional (3D) structural representation of $\mathrm{TB}_{3}$ molecule 


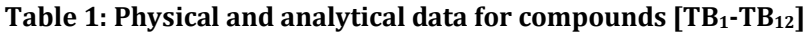

\begin{tabular}{|c|c|c|c|c|c|c|}
\hline Entry & $\mathbf{A r}$ & Mol.formula & Mol. wt & Melting point $\left({ }^{\circ} \mathrm{C}\right)$ & $\mathbf{R}_{\mathbf{f}}$ & \% Yield \\
\hline $\mathrm{TB}_{1}$ & $\mathrm{C}_{6} \mathrm{H}_{5}$ & $\mathrm{C}_{25} \mathrm{H}_{28} \mathrm{~N}_{2} \mathrm{~S}$ & 388.56 & $184-186$ & 0.71 & 95 \\
\hline $\mathrm{TB}_{2}$ & $\mathrm{P}-\mathrm{ClC}_{6} \mathrm{H}_{4}$ & $\mathrm{C}_{25} \mathrm{H}_{26} \mathrm{Cl}_{2} \mathrm{~N}_{2} \mathrm{~S}$ & 457.45 & $222-224$ & 0.78 & 80 \\
\hline $\mathrm{TB}_{3}$ & $\mathrm{P}-\mathrm{FC}_{6} \mathrm{H}_{4}$ & $\mathrm{C}_{25} \mathrm{H}_{26} \mathrm{~F}_{2} \mathrm{~N}_{2} \mathrm{~S}$ & 424.54 & $243-245$ & 0.65 & 82 \\
\hline $\mathrm{TB}_{4}$ & $4-(\mathrm{OMe}) \mathrm{C}_{6} \mathrm{H}_{4}$ & $\mathrm{C}_{27} \mathrm{H}_{32} \mathrm{~N}_{2} \mathrm{O}_{2} \mathrm{~S}$ & 448.62 & $189-192$ & 0.48 & 89 \\
\hline $\mathrm{TB}_{5}$ & $\mathrm{P}-\mathrm{CH}(\mathrm{Me})_{2} \mathrm{C}_{6} \mathrm{H}_{4}$ & $\mathrm{C}_{31} \mathrm{H}_{40} \mathrm{~N}_{2} \mathrm{~S}$ & 472.72 & $180-182$ & 0.56 & 88 \\
\hline $\mathrm{TB}_{6}$ & $3,4,-(\mathrm{OMe})_{2} \mathrm{C}_{6} \mathrm{H}_{3}$ & $\mathrm{C}_{29} \mathrm{H}_{36} \mathrm{~N}_{2} \mathrm{O}_{4} \mathrm{~S}$ & 508.67 & $166-168$ & 0.71 & 78 \\
\hline $\mathrm{TB}_{7}$ & $3,4,5-(\mathrm{OMe}){ }_{3} \mathrm{C}_{6} \mathrm{H}_{2}$ & $\mathrm{C}_{31} \mathrm{H}_{40} \mathrm{~N}_{2} \mathrm{O}_{6} \mathrm{~S}$ & 568.72 & $210-212$ & 0.54 & 94 \\
\hline $\mathrm{TB}_{8}$ & $\mathrm{P}-\mathrm{N}(\mathrm{Me}){ }_{2} \mathrm{C}_{6} \mathrm{H}_{4}$ & $\mathrm{C}_{29} \mathrm{H}_{38} \mathrm{~N}_{4} \mathrm{~S}$ & 474.70 & $142-144$ & 0.76 & 69 \\
\hline $\mathrm{TB}_{9}$ & $\mathrm{P}-\mathrm{NH}_{2} \mathrm{C}_{6} \mathrm{H}_{4}$ & $\mathrm{C}_{25} \mathrm{H}_{30} \mathrm{~N}_{4} \mathrm{~S}$ & 418.59 & $156-158$ & 0.69 & 70 \\
\hline $\mathrm{TB}_{10}$ & $\mathrm{P}-\mathrm{C}_{2} \mathrm{H}_{5} \mathrm{C}_{6} \mathrm{H}_{4}$ & $\mathrm{C}_{29} \mathrm{H}_{36} \mathrm{~N}_{2} \mathrm{~S}$ & 444.67 & $178-180$ & 0.66 & 76 \\
\hline $\mathrm{TB}_{11}$ & $\mathrm{P}-\mathrm{NO}_{2} \mathrm{C}_{6} \mathrm{H}_{4}$ & $\mathrm{C}_{25} \mathrm{H}_{26} \mathrm{~N}_{4} \mathrm{O}_{4} \mathrm{~S}$ & 478.56 & $182-184$ & 0.78 & 65 \\
\hline $\mathrm{TB}_{12}$ & $2,4-(\mathrm{OH})_{2} \mathrm{C}_{6} \mathrm{H}_{5}$ & $\mathrm{C}_{25} \mathrm{H}_{28} \mathrm{~N}_{2} \mathrm{O}_{4} \mathrm{~S}$ & 452.56 & $177-178$ & 0.52 & 65 \\
\hline
\end{tabular}

Ar-aromatic, retardation factor $\left(\mathrm{R}_{\mathrm{f}}\right)$, TLC solvent system(x: y) n-hexane and ethyl acetate (8:2)

\section{Biological evaluation}

\section{Acute oral toxicity studies}

Maximum tolerated dose (MTD) study was carried out as per the Organization of Economical Co-Operation and Development (OECD) guidelines $423[31,32]$. All the synthesized compounds treated animals, showed only mounting and genital licking to a mild degree while all the other responses were normal when compared to control at $24 \mathrm{hr}$ observation. The 48 and $72 \mathrm{hr}$ observation revealed no changes in any of the normal behavioural pattern of the treated groups to that of the control group. No toxicity or death was observed for all the compounds on the administration of a dose of $2000 \mathrm{mg} / \mathrm{kg}$. Hence, all those compounds can be considered as safe (X-classify). A group of three albino mice of either sex weighing 18-30 gm and of $90 \mathrm{~d}$ age selected randomly and used for acute toxicity studies. The animals were kept observed fasting overnight, prior to the experimental procedure. The technique up and down or staircase was used to establish the dose. The synthesised compounds were administered orally at the dose level of 5 $\mathrm{mg} / \mathrm{kg}$ body weight to the animals and observed for $14 \mathrm{~d}$. The investigational protocol for the pharmacological selection was done in accordance, with the guidelines prescribed by an Institutional Animal Ethics Committee (CPCSEA No: 1292/ac/09/CPCSEA). Since no mortality was observed, the procedure has been repeated for further higher doses of 50, 300 and $2000 \mathrm{mg} / \mathrm{kg}$ body weight. The compounds show no mortality at a dose up to $2000 \mathrm{mg} / \mathrm{kg}$. Hence 1/10th (200 $\mathrm{mg} / \mathrm{kg})$ and $1 / 5 \mathrm{th}(400 \mathrm{mg} / \mathrm{kg})$ of this dose were selected as the dose levels for the study.

\section{Pentylenetetrazole (PTZ) induced seizure model General procedure}

Adult male mice with a body weight between $18 \mathrm{~g}$ and $22 \mathrm{~g}$ were used. The test compounds and the standard drug, diphenyl hydantain (30 $\mathrm{mg} / \mathrm{kg}$ ) were given orally to a group of 6 mice. Another group of 6 mice served as control, after $60 \mathrm{~min}$, oral administration, $80 \mathrm{mg} / \mathrm{kg}$ PTZ was injected through intra-peritoneal route. Each animal was placed into an individual plastic cage, and the animals were observed for $30 \mathrm{~min}$ for tonic convulsion episode. Hind limb extension was taken as a tonic convulsion. Latency of convulsion, $\%$ of convulsion, time of death, $\%$ of survival was recorded. The number of animals protected from hind limb tonic extension seizures (HLTE) and the time spent in this position were determined, for each dose group (mean \pm SEM) in PTZ induced method. The onset of tonic convulsions and the number of animals convulsing, or not convulsing within the observation period were noted. The ability of the test compounds to prevent or delay the onset of the hind limb extension exhibited by the animals was taken as an indication of anticonvulsant activity.

\section{Computationally predicted toxicity, lipophilicity and drug score} profiles

The shredding of each molecule at every rotatable bond leads to a set of nucleus fragments. These, in turn, were used to rebuild all possible larger fragments which could be the substructure of the original fragment. Afterwards, a search procedure for substructure resolute the occurrence, frequency of every one of the fragment (constructed and core fragments) surrounded by all traded drugs of 3300 in addition to 15,000 commercially available chemicals (Fluka) to predict toxicity, C $\log \mathrm{P}$, and drug score $[33,34]$.

\section{In vitro antimicrobial activity}

\section{Antibacterial activity}

In vitro antibacterial activity of the newly synthesized compounds were tested against the standard cultures of gram-positive organisms, such as Staphylococcus aureus and Klebsiella pneumoniae and gram-negative organisms like Escherichia coli and Pseudomonas aeruginosa by cup plate agar diffusion method. Bacteria were cultured in nutrient agar medium, and the solutions of the synthesized compounds were dissolved in dimethyl sulfoxide (DMSO $1 \mathrm{ml}$ ) at $150 \mu \mathrm{g} / \mathrm{ml}$ concentration and the compounds incubated at $37^{\circ} \mathrm{C}$ for $24 \mathrm{hr}$. After the incubation period, the inhibition zones were measured in $\mathrm{mm}$, and the known antibiotics like ciprofloxacin at a concentration of $10 \mu \mathrm{g} / \mathrm{ml}$ as a standard drug were used for the comparison at the same concentration [35, 36]. The results are tabulated in the table 2 .

Table 2: Antimicrobial activity of the compounds on bacterial and fungal strains

\begin{tabular}{|c|c|c|c|c|c|c|}
\hline \multirow[t]{2}{*}{ compounds } & \multicolumn{6}{|c|}{ Antimicrobial activity in terms of zone of inhibition in millimeter (mm) } \\
\hline & S. aureus & K. pneamoniae & E. coli & P. aeruginosa & C. albicans & A. niger \\
\hline $\mathrm{TB}_{1}$ & $15 \pm 0.12$ & $14 \pm 1.21$ & $16 \pm 0.15$ & $14 \pm 0.85$ & $15 \pm 0.31$ & $12 \pm 0.65$ \\
\hline $\mathrm{TB}_{2}$ & $14 \pm 0.64$ & $13 \pm 0.82$ & $15 \pm 0.66$ & $13 \pm 0.16$ & $16 \pm 0.54$ & $13 \pm 1.76$ \\
\hline $\mathrm{TB}_{3}$ & $13 \pm 1.72$ & $15 \pm 0.34$ & $14 \pm 1.58$ & $15 \pm 0.88$ & $15 \pm 1.06$ & $14 \pm 0.86$ \\
\hline $\mathrm{TB}_{4}$ & $16 \pm 0.51$ & $15 \pm 0.11$ & $15 \pm 0.60$ & $14 \pm 1.59$ & $17 \pm 0.53$ & $15 \pm 0.64$ \\
\hline $\mathrm{TB}_{5}$ & $15 \pm 0.42$ & $16 \pm 1.54$ & $16 \pm 0.02$ & $16 \pm 0.07$ & $16 \pm 0.64$ & $16 \pm 0.56$ \\
\hline $\mathrm{TB}_{6}$ & $14 \pm 0.41$ & $14 \pm 0.03$ & $15 \pm 1.72$ & $15 \pm 0.46$ & $14 \pm 0.13$ & $12 \pm 0.25$ \\
\hline $\mathrm{TB}_{7}$ & $12 \pm 0.83$ & $13 \pm 0.12$ & $13 \pm 0.13$ & $12 \pm 0.19$ & $14 \pm 0.37$ & $14 \pm 1.73$ \\
\hline $\mathrm{TB}_{8}$ & $13 \pm 1.62$ & $11 \pm 0.04$ & $13 \pm 0.95$ & $14 \pm 1.16$ & $13 \pm 0.68$ & $13 \pm 0.54$ \\
\hline $\mathrm{TB}_{9}$ & $15 \pm 0.64$ & $16 \pm 1.61$ & $15 \pm 0.67$ & $16 \pm 1.52$ & $12 \pm 1.86$ & $16 \pm 0.46$ \\
\hline $\mathrm{TB}_{10}$ & $16 \pm 1.14$ & $15 \pm 0.81$ & $17 \pm 0.01$ & $18 \pm 0.72$ & $15 \pm 0.47$ & $13 \pm 0.41$ \\
\hline $\mathrm{TB}_{11}$ & $14 \pm 1.53$ & $12 \pm 0.19$ & $14 \pm 0.86$ & $13 \pm 0.18$ & $12 \pm 0.79$ & $15 \pm 0.08$ \\
\hline $\mathrm{TB}_{12}$ & $16 \pm 0.91$ & $13 \pm 1.02$ & $13 \pm 1.52$ & $14 \pm 0.71$ & $14 \pm 0.67$ & $15 \pm 0.53$ \\
\hline Ciprofloxacin & $22 \pm 0.64$ & $25 \pm 0.03$ & $22 \pm 0.17$ & $25 \pm 0.64$ & ---------- & --------. \\
\hline Ketaconazole & 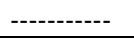 & --------- & --------- & --------- & $24 \pm 1.89$ & $23 \pm 0.85$ \\
\hline
\end{tabular}

Diameter of zone of inhibition results are expressed as mean \pm SD. Data compared against positive standard group and each test group. 


\section{Antifungal activity}

The antifungal activity of the synthesized compounds was evaluated against two fungal organisms Candida albicans and Asperigillus niger by using cup plate agar diffusion method and inoculated at $28 \pm 1{ }^{\circ} \mathrm{C}$ for seven days. After seven days, inhibition in fungal growth was resolute as a difference in growth between control plates and those treated with test compound and compared with the standard drug ketaconazole at a concentration of $20 \mu \mathrm{g} / \mathrm{ml}$ and the results were tabulated in the table 2 . The observed zone of inhibition and the minimum inhibitory concentration (MIC) values for all the synthesized compounds are to be had in table 3 . All the tested compounds have showed optimum antimicrobial activity with minor differences in their zone of inhibitions when compared with the standard drugs.

\section{Minimum inhibitory concentration (MIC)}

Minimum inhibitory concentration (MIC), anti-microbial activity of each compound was evaluated at the concentration of $150 \mu \mathrm{g} / \mathrm{ml}$; values are expressed as mean $\pm S D$. Data compared against the positive standard group.

Table 3: Minimum inhibitory concentration (MIC) data of the synthesized compounds

\begin{tabular}{|c|c|c|c|c|c|c|}
\hline \multirow[t]{2}{*}{ Entry } & \multicolumn{6}{|c|}{ Diameter of zone of inhibition in millimeter (MIC) $\mu \mathrm{g} / \mathrm{ml}$} \\
\hline & S. aureus & K. pneamoniae & E. coli & P. aeruginosa & C. albicans & A. niger \\
\hline $\mathrm{TB}_{1}$ & $21 \pm 0.12^{* *}$ & $20 \pm 0.42$ & $18 \pm 0.11$ & $18 \pm 0.65$ & $23 \pm 0.18^{* *}$ & $22 \pm 0.75^{*}$ \\
\hline $\mathrm{TB}_{2}$ & $22 \pm 0.03^{* *}$ & $19 \pm 0.64$ & $16 \pm 0.63$ & $17 \pm 0.88$ & $22 \pm 0.82^{*}$ & $21 \pm 0.36$ \\
\hline $\mathrm{TB}_{3}$ & $19 \pm 0.11$ & $19 \pm 0.72$ & $18 \pm 0.52$ & $16 \pm 1.45$ & $22 \pm 0.48^{*}$ & $23 \pm 1.75^{*}$ \\
\hline $\mathrm{TB}_{4}$ & $21 \pm 0.13$ & $20 \pm 0.83$ & $18 \pm 0.71$ & $17 \pm 1.84$ & $22 \pm 0.51 *$ & $22 \pm 0.59 *$ \\
\hline $\mathrm{TB}_{5}$ & $24 \pm 0.17 * * *$ & $23 \pm 0.12^{* *}$ & $21 \pm 0.42^{* *}$ & $22 \pm 0.54^{* *}$ & $25 \pm 0.01^{* *}$ & $26 \pm 0.64^{* * *}$ \\
\hline $\mathrm{TB}_{6}$ & $19 \pm 0.61$ & $20 \pm 1.84$ & $19 \pm 0.67$ & $16 \pm 0.17$ & $21 \pm 0.50$ & $18 \pm 0.18$ \\
\hline $\mathrm{TB}_{7}$ & $18 \pm 1.02$ & $22 \pm 0.73^{* *}$ & $16 \pm 0.91$ & $17 \pm 0.64$ & $23 \pm 1.63$ & $22 \pm 0.86^{*}$ \\
\hline $\mathrm{TB}_{8}$ & $21 \pm 0.41^{* *}$ & $20 \pm 0.92$ & $18 \pm 0.12$ & $16 \pm 0.42$ & $22 \pm 0.45$ & $24 \pm 0.61^{* *}$ \\
\hline $\mathrm{TB}_{9}$ & $19 \pm 0.53$ & $16 \pm 0.71$ & $14 \pm 0.50$ & $15 \pm 0.78$ & $21 \pm 0.14$ & $19 \pm 0.45$ \\
\hline $\mathrm{TB}_{10}$ & $19 \pm 0.74$ & $18 \pm 1.32$ & $17 \pm 1.81$ & $16 \pm 0.53$ & $24 \pm 1.20^{* *}$ & $21 \pm 1.65$ \\
\hline $\mathrm{TB}_{11}$ & $21 \pm 1.21^{* *}$ & $14 \pm 1.71$ & $16 \pm 1.49$ & $16 \pm 0.17$ & $22 \pm 1.64$ & $20 \pm 1.57$ \\
\hline $\mathrm{TB}_{12}$ & $19 \pm 0.95$ & $21 \pm 0.81^{* *}$ & $18 \pm 0.62$ & $18 \pm 0.64$ & $23 \pm 0.36^{* *}$ & $21 \pm 0.43$ \\
\hline Ciprofloxacin & $26 \pm 0.65^{* * *}$ & $26 \pm 0.79 * * *$ & $24 \pm 0.99 * * *$ & $24 \pm 1.21^{* * *}$ & - & - \\
\hline Ketaconazole & 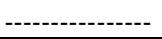 & - & 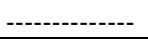 & 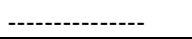 & $28 \pm 0.28 * * *$ & $28 \pm 0.04^{* * *}$ \\
\hline
\end{tabular}

Diameter of the zone of inhibition for minimum inhibitory concentration results are expressed as meantstandard deviation of triplicates. The diameter of well plates was $8 \mathrm{~mm}$, Ciprofloxacin $10 \mu \mathrm{g} / \mathrm{ml}$ used as standard against Staphylococcus aureus, Klebsiella pneumoniae, Escherichia coli, and Pseudomonas aeruginosa, Ketaconazole at a concentration of $20 \mu \mathrm{g} / \mathrm{ml}$ was used as standard against Candida albicans and Asperigillus niger

\section{RESULTS AND DISCUSSION}

\section{Chemistry}

Twelve novel 1, 3-thiazine derivatives $\left(\mathrm{TB}_{1}-\mathrm{TB}_{12}\right)$ were synthesized by the condensation of different 2, 6-dibenzylidene-4-tert-butylcyclohexanones with thiourea in the presence of ethanol by refluxation. The yields of the compounds were in between $65-94 \%$. All the compounds were purified by column chromatography and characterized by spectral methods including IR, ${ }^{1} \mathrm{H}$ NMR, ${ }^{13} \mathrm{C}$ NMR, and Mass. Formation of 1,3-thiazine scaffold is confirmed by the characteristic IR absorption bands in the ranges of $3250-3450 \mathrm{~cm}^{-1}$ (imine NH-), 2950-3200 (cyclic NH-), 1590-1680 (C=N) and 10201120 (C-N) respectively.

Two diagnostic singlet peaks in the ${ }^{1} \mathrm{H}$ NMR spectrums around $\delta 6.4$ $7.2 \mathrm{ppm}$ helped to unravel the structures of the compounds. The ${ }^{13} \mathrm{C}$ NMR spectrum of compounds exhibited the characteristic signals in the range of $\delta 170-190 \mathrm{ppm}$ which corresponds to $\mathrm{C}=\mathrm{N}$, apart from the peaks corresponding to the other carbons. The mass spectra obtained by positive mode of ionization method revealed that the $[\mathrm{M}+\mathrm{H}]^{+}$ions are representing the molecular weight of the compound.
The results of elemental analysis were also in close agreement within $\pm 0.4 \%$ of the calculated values.

\begin{abstract}
Anticonvulsant activity
1, 3-thiazine derivatives were tested for their anticonvulsant activity by pentylenetetrazole (PTZ) induced seizures. The results are summarized in table 4 . From the results it can be clearly observed that 1,3-thiazines possess significant anticonvulsant activity, but less than the standard diphenyl hydantain. Compound $\mathrm{TB}_{7}$ containing 3, 4, 5-trimethoxyphenyl moiety was the most potent of the sequence with the onset of convulsions by $18.7 \mathrm{~min}$. This is followed by $\mathrm{TB}_{5}$ with 4-isopropylphenyl substitution with $14.1 \mathrm{~min}$.

The third and fourth in the activity are seen, in $\mathrm{TB}_{6}$ with 3, 4dimethoxyphenyl with 11.7 min and $\mathrm{TB}_{4}$ with 4-methoxyphenyl with onset 11.3 min scaffolds respectively. Compound $\mathrm{TB}_{10}$ with 4ethylphenyl moiety with $10.7 \mathrm{~min}$ also possess significant anticonvulsant activity. Halogenated thiazines were also exhibited superior activity, and other compounds have shown moderate to mild antiepileptic activity. However, none of these compounds has greater activity than the standard diphenyl hydantain.
\end{abstract}

Table 4: Hind limb tonic extension seizure (HLTE)

\begin{tabular}{lll}
\hline Groups & Latency of onset of convulsion (in min) & Time of death* (in min) \\
\hline Control (Normal saline) & $1.071 \pm 0.048$ & $5.72 \pm 0.3116$ \\
Standard (Diphenyl hydantain) & $28.31 \pm 0.341$ & Prevented \\
TB & $5.122 \pm 0.187^{*}$ & $10.62 \pm 2.668^{*}$ \\
TB & $11.36 \pm 0.3411^{* *}$ & $25.48 \pm 2.857^{* * *}$ \\
TB1 & $1.375 \pm 0.1661$ & $9.77 \pm 1.058$ \\
TB5 & $14.15 \pm 0.321^{* * *}$ & $25.27 \pm 0.523^{* * *}$ \\
TB7 & $18.73 \pm 0.1241^{* * *}$ & $30.87 \pm 0.506^{* * *}$ \\
TB6 & $11.71 \pm 0.182^{* *}$ & $15.82 \pm 0.512^{*}$ \\
TB10 & $10.76 \pm 0.192^{* *}$ & $16.62 \pm 0.612^{*}$ \\
\hline
\end{tabular}

Stastical standard: ${ }^{*} \mathrm{P}<0.1,{ }^{* *} \mathrm{P}<0.01 ;{ }^{* * *} \mathrm{P}<0.001$, Time of death: 30 min was taken as the cut off time all values are mean \pm SEM values using $\mathrm{n}=6$ animals in each group. 


\section{Statistics}

The statistical analysis of the obtained results from diphenyl hydantain and the synthesized compounds were made. The significance of differences between the different dosing groups were calculated by the Steel test (seizure score), by one-way analysis of variance (ANOVA) followed by post hoc comparison using Dunnett's test, $p<0.05$ was considered statistically significant.

\section{In vitro antimicrobial activity}

All the newly synthesized compounds were tested for their antimicrobial activity against two gram-positive (Staphylococcus aures and Klebisiella pneamoniae), two gram negative (Escherichia coli and Pseudomonas aeruginosa) bacterial and two fungal strains (Candida albicans and Asperigillus niger) at a concentration of 150 $\mu \mathrm{g} / \mathrm{ml}$ using cup plate method and minimum inhibitory concentration (MIC) was determined by agar streak dilution method. From the results, we have observed that $\mathrm{TB}_{5}$ have been shown best antimicrobial activity in all the bacterial and fungal strains, $\mathrm{TB}_{2}$ also shown better activity, the rest of the compounds have been shown good antimicrobial activity. $\mathrm{TB}_{3}$ substituted moiety (fig. 2) have been shown potent antifungal activity.

\section{Molecular docking and scoring function}

\section{Docking protocol and their validation}

Molecular modeling studies were performed on workstation running Red Hat enterprise and Linux 4.0 and selection of the compounds depends on the compounds which are obeying Lipinsky rule of five and used an automated docking software Glide 5.6
(Schrodinger-Maestro) that applies a two stage scoring process to sort out the best conformations and orientations of the ligand (defined as pose), based on its interaction pattern with the receptor, docked at its active site using a standard docking protocol. The newly synthesised novel compounds $\mathrm{TB}_{1}$ to $\mathrm{TB}_{12}$ were selected for performing molecular docking studies. Molecules were built using Maestro build panel and prepared by LigPrep 2.0 application. Crystal structures of $S$. aureus gyrase complex with ciprofloxacin and deoxyribonucleic acid (DNA), protein data bank (PDB id: 2XCT) [37], and structures of cytochrome P450 family 51 subfamily A member 1 [CYP51] from the pathogens Candida glabrata (PDB id: 5JLC) were downloaded from protein data bank (www. rcsb. org). GLIDE 5.6 was used for molecular docking. The proteins were prepared using protein preparation module applying the default parameters. The grid was generated around the active site of the protein by selecting the cocrystalized ligand. Receptor vander Waals scaling for the nonpolar atoms was kept 0.9 [38], the Low-energy conformation of the ligands was selected and docked into the grid using standard precision (SP) docking mode. Dock pose of each ligand was analyzed for interactions with the receptor.

Computer-assisted drug design (CADD) approach has contributed to the discovery of several novel drugs. Molecular docking continues to hold great promise in the field of computer-based drug design. In silico docking, scores are provided in table 5 . Sources from protein data bank: 2XCT, 5JLC both are IDs for docking of antimicrobial activity. 2XCT: The twinned 3.35 a structure of $s$. aureus gyrase complex with ciprofloxacin and DNA, classification: Isomerase/DNA/ antibiotic. 5JLC: Structure of CYP51 from the pathogen Candida glabrata, classification: oxidoreductase/ oxidoreductase inhibitor.

Table 5: Docking score of $\mathrm{TB}_{1}-\mathrm{TB}_{12}$ molecules from SP docking protocol in Glide 5.6

\begin{tabular}{llll}
\hline S. No. & Molecule & DNA gyrase PDB ID: 2XCT & CYP51A1 PDB ID: 5JLC \\
\hline 1 & TB1 & -9.05 & -8.72 \\
2 & TB2 & -8.42 & -9.76 \\
3 & TB3 & -8.622 & -8.92 \\
4 & TB4 & -8.15 & -9.24 \\
5 & TB5 & -7.98 & -10.79 \\
6 & TB6 & -8.22 & -7.98 \\
7 & TB7 & -4.66 & -3.86 \\
8 & TB8 & -7.85 & -9.81 \\
9 & TB9 & -8.12 & -9.61 \\
10 & TB10 & -8.17 & -10.63 \\
11 & TB11 & -8.74 & -7.69 \\
12 & TB12 & -8.12 & -9.77 \\
13 & Ciproflaxin & -12.21 & --- \\
14 & Ketaconazole & --- & -9.76 \\
\hline
\end{tabular}

Molecular docking of the drug molecule into the binding site of target receptor gives important information about drug-receptor interactions and is frequently used to find out the binding mode of drug candidates to their protein targets in order to predict the affinity. Molecular docking studies of $\mathrm{TB}_{1}-\mathrm{TB}_{12}$ were carried out on DNA gyrase and CYP51A1 protein targets.

\section{DNA gyrase}

DNA gyrase is an enzyme that relieves strain, while double-strand DNA is being unwound by helicase. This causes negative supercoiling of the DNA. Bacterial DNA gyrase supercoils into DNA by looping the template so as to form a crossing, then cutting one of the double helices and passing the other through it before releasing the break, changing the linking number by two in each enzymatic step [39]. Bacterial DNA gyrase is the target of many antibiotics, including ciprofloxacin that binds to this enzyme and prevents it from decatenation replicating DNA.

Molecular docking and dock pose analysis of $\mathrm{TB}_{1}-\mathrm{TB}_{12}$ molecules into DNA gyrase (PDB id: 2XCT) showed similar interaction as that of standard drug ciprofloxacin. $\mathrm{TB}_{1}$ had the highest binding affinity in terms of dockscore- $9.05 \mathrm{kcal} / \mathrm{mol}$. The molecule showed hydrogen bond interaction with Ser1084 amino acid and $\mathrm{G}_{8}$ nucleotide from DNA, hydrophobic interactions with $\mathrm{G}_{8}, \mathrm{C}_{12}, \mathrm{C}_{13}$ nucleotides and Arg 458 amino acid residues. Fig. 3 shows the binding pose of $\mathrm{TB}_{1}$ in DNA gyrase along with intermolecular interactions.
CYP51 A1 (Lanosterol $14 \alpha$-demethylase) is a cytochrome P450 enzyme and converts Lanosterol to 4, 4-dimethylcholesta-8(9), 14,

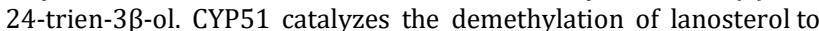
create a key precursor that is converted into ergosterol in fungi [40]. Antifungal azoles like ketaconazole target CYP51 activity and prevent the production of this key compound [41]. Molecular docking and dock pose analysis of $\mathrm{TB}_{1}-\mathrm{TB}_{12}$ molecules into CYP51A1 (PDB id: 5JLC) showed good dock score indicating better binding. $\mathrm{TB}_{5}$ had the highest binding affinity in terms of dockscore-10.79 $\mathrm{kcal} / \mathrm{mol}$. The molecule showed hydrogen bond interaction with Met 512 amino acid, hydrophobic interactions with Heme group and Tyr 73, Tyr121, Tyr 141, Met 512 and Phe 242 amino acid residues. Figs.

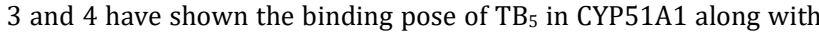
intermolecular interactions.

\section{Assessment of toxicity, lipophilicity and drug score profiles}

Osiris program was used for the prophecy of the toxicity of the synthesized compounds. The prediction relies on a substructure look for route determining the occurrence frequency of any fragment (constructed and core fragments) within any of toxicity 
classes. The drug score combines $\mathrm{C} \log \mathrm{P}$, molecular weight and toxicity risks in one handy value that may be used to judge the compounds overall potential to meet the requirements for a drug. Osiris program was used for the prophecy of the $C \log P$ of compounds. $\mathrm{C} \log \mathrm{P}$ is a well-established parameter to determine the hydrophilicity of the synthesised compounds. Compounds show a reasonable probability of being well absorbed when they have $C \log$ $P$ value around 5.0. All synthesized 1, 3-thiazin-2-imine derivatives have showed low Insilco possible toxicity risks. From the table, it was observed that all compounds had revealed $\mathrm{C} \log \mathrm{P}$ around 5.0 indicating that the synthesized compounds could be potential drug candidates. Prophecy of $\mathrm{C} \log \mathrm{P}$, drug score and toxicity for 1 , 3thiazin-2-imine derivatives are prearranged in table 6 , and almost all the compounds possess good values of drug score, $C \log \mathrm{P}$, and low probable toxicity risks as revealed by computational in silico studies and the values are tabulated in table 6 .

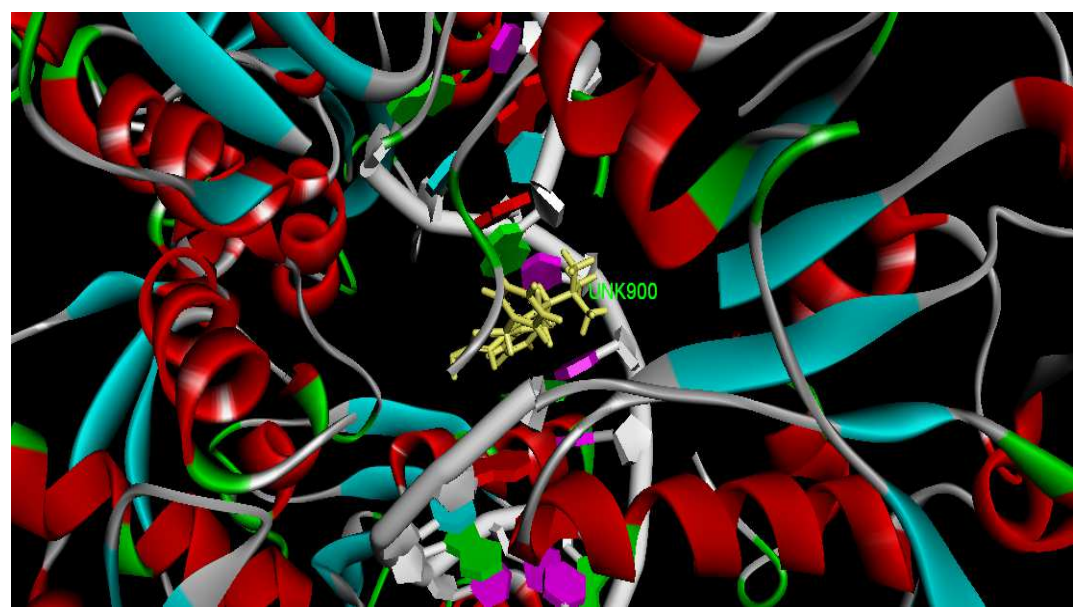

3 Dimensional (3D) structure image of $\mathrm{TB}_{1}$ in DNA gyrase [a]

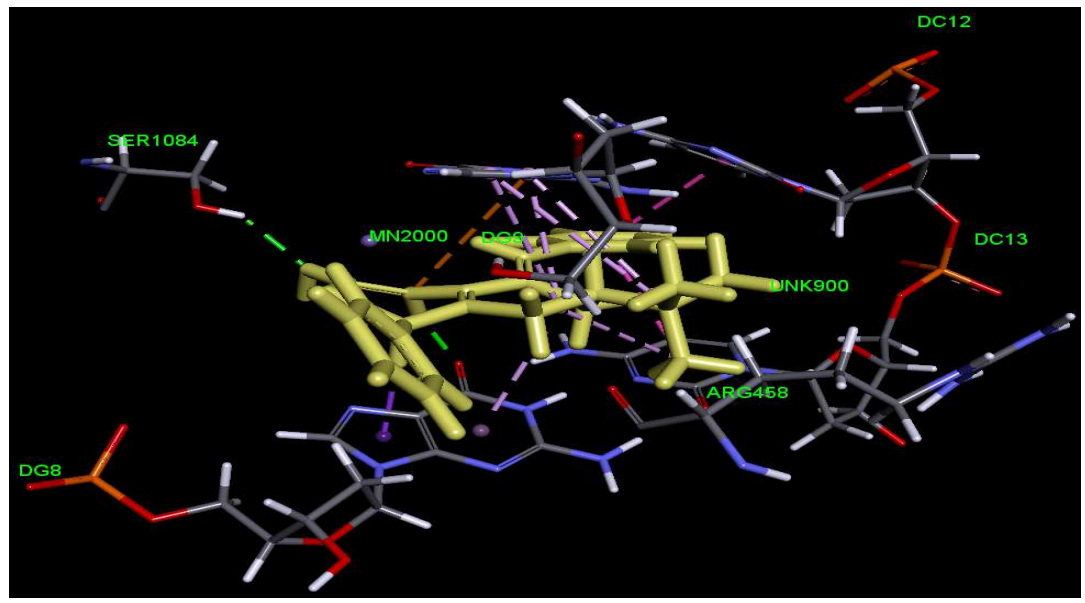

3 Dimensional (3D) structure image dockpose of $\mathrm{TB}_{1}$ showing hydrogen bond [b]

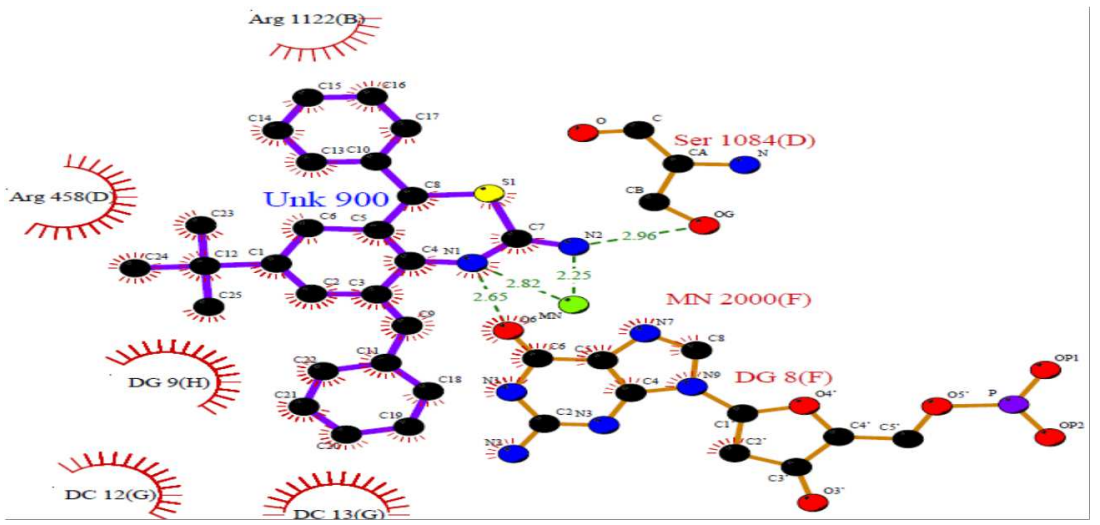

Graphical (2 Dimensional) image of $T B_{1}$ showing ligand-receptor interaction[c]

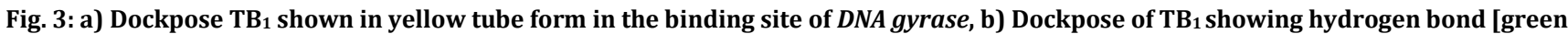
line] and hydrophobic pi-pi and pi-alkyl interaction [pink and light pink lines], c) Two dimensional Ligplot obtained from PDBsum [www.ebi.ac.uk] of TB 1 showing ligand-receptor interaction CYP51A1 


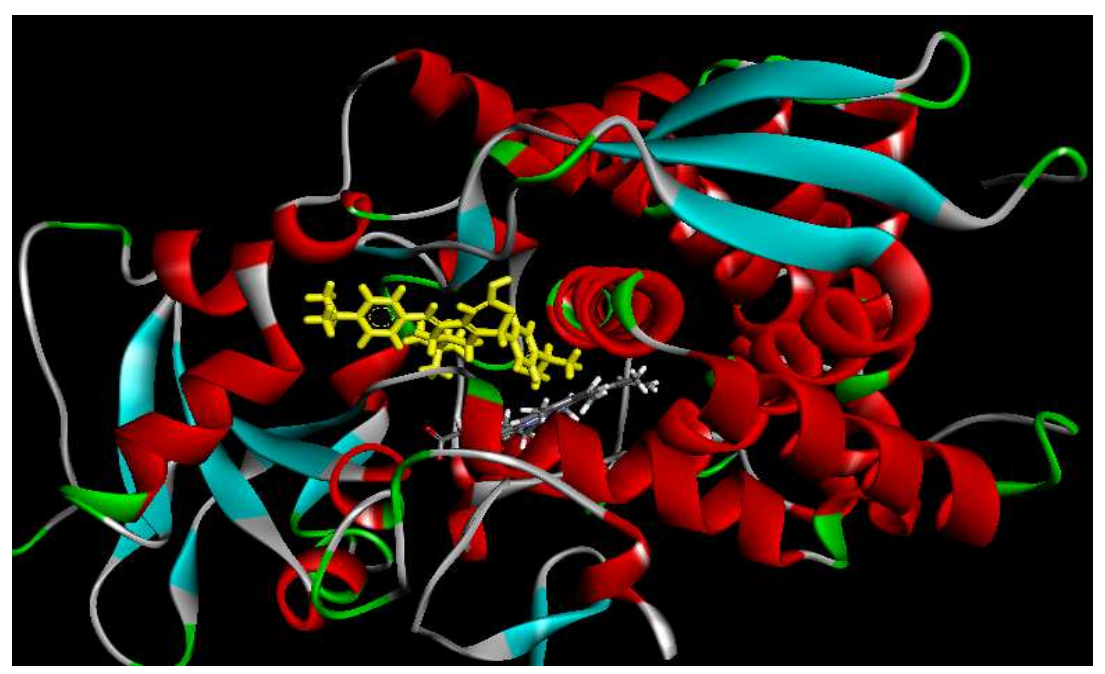

3 Dimensional (3D) structure image of $\mathrm{TB}_{5}$ in CYP51A1 [a]

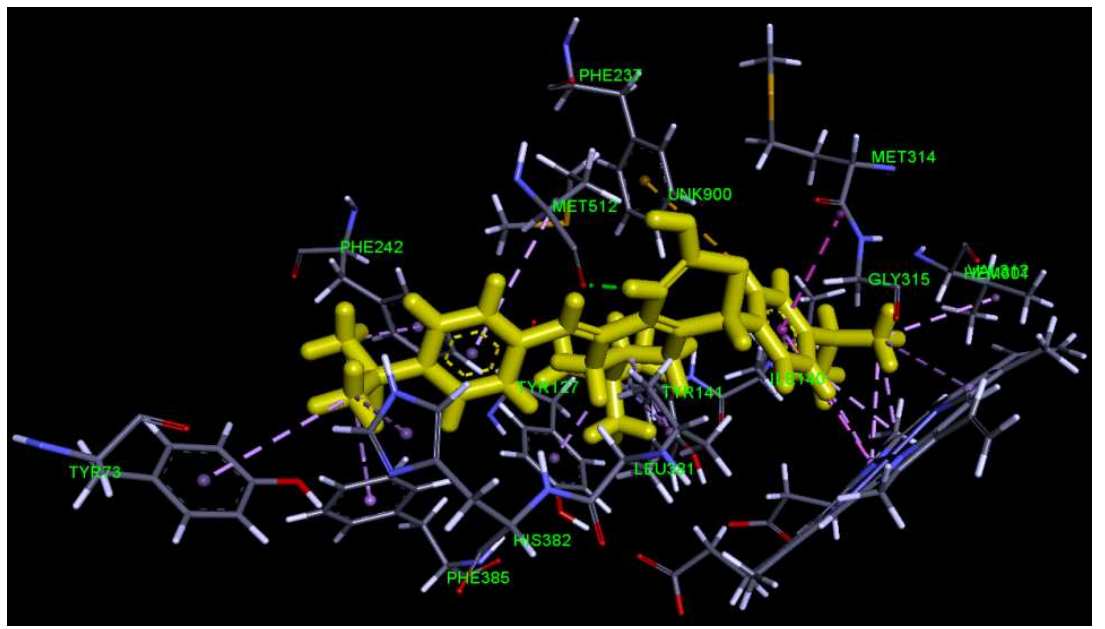

3 Dimensional (3D) structure image dockpose of $\mathrm{TB}_{5}$ showing hydrogen bond [b]

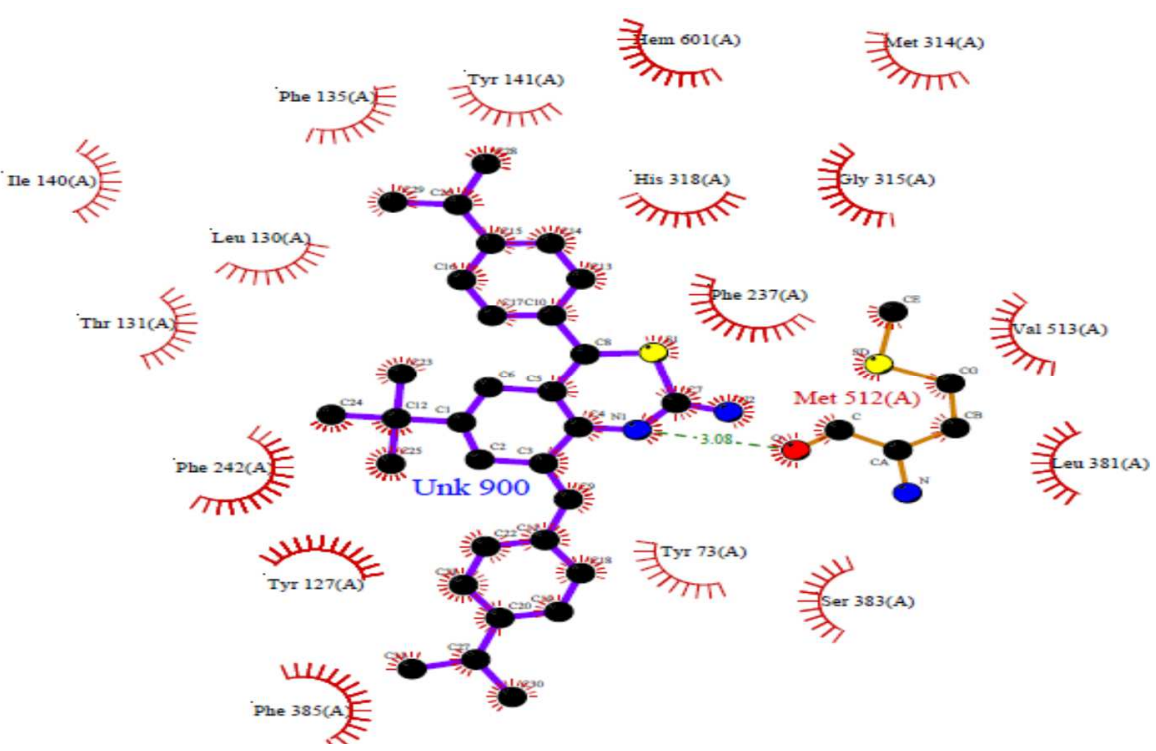

Graphical 2 Dimensional (2D) image of $\mathrm{TB}_{5}$ showing ligand-receptor interaction [c]

Fig. 4: a) Dockpose $\mathrm{TB}_{5}$ shown in yellow tube form in the binding site of CYP51A1, b) Dockpose of $\mathrm{TB}_{5}$ showing hydrogen bond [green line] and hydrophobic pi-pi and pi-alkyl interaction [pink and light pink lines], c) Two dimensional ligplot obtained from PD Bsum [www.ebi.ac.uk] of $\mathrm{TB}_{5}$ showing the ligand-receptor interaction 
Table 6: Computationally predicted toxicity risks, lipophilicity drug scores

\begin{tabular}{llll}
\hline Entry & Clog $\mathbf{P}^{*}$ & Drug score range & Toxicity risks** \\
\hline $\mathrm{TB}_{1}$ & 6.12 & 0.27 & Negative \\
$\mathrm{TB}_{2}$ & 7.35 & 0.25 & Negative \\
$\mathrm{TB}_{3}$ & 6.24 & 0.21 & Negative \\
$\mathrm{TB}_{4}$ & 5.91 & 0.37 & Negative \\
$\mathrm{TB}_{5}$ & 8.31 & 0.08 & Negative \\
$\mathrm{TB}_{6}$ & 5.38 & 0.33 & Negative \\
$\mathrm{TB}_{7}$ & 5.49 & 0.31 & Negative \\
$\mathrm{TB}_{8}$ & 5.53 & 0.37 & Negative \\
$\mathrm{TB}_{9}$ & 4.93 & 0.42 & Negative \\
$\mathrm{TB}_{10}$ & 7.47 & 0.21 & Negative \\
\hline
\end{tabular}

${ }^{*}$ Indicating Clog P values calculated for the lipophilicity, ${ }^{* *}$ Indicating the mutagenicity, tumorigenicity, irritancy and reproductive effects

\section{CONCLUSION}

In conclusion, the results of the present study indicated that the synthesized compounds have striking anticonvulsant as well as antimicrobial activities. In the case of antimicrobial activity, among all the compounds from $\mathrm{TB}_{1}-\mathrm{TB}_{12}$ isopropyl derivative have shown better activity, whereas the other compounds were shown potent activity, in the case of antifungal activity, along with that ethyl and dihydroxy derivative shown high antifungal activity. From pentylene-tetrazole (PTZ) induced model it can be clearly notified that 1,3-thiazines possess significant anticonvulsant activity but less than the standard diphenyl hydantain, moreover anticonvulsant activity in pentylenetetrazole (PTZ) induced seizures, identifies compound that can raise seizure threshold in the brain [42], pentylenetetrazole (PTZ) has been shown to act together with gamma-amino-butyric acid (GABA) neurotransmitters and the GABA receptor complex. The compound $\mathrm{TB}_{7}$ containing $3,4,5-$ trimethoxyphenyl substituents on the thiazine moiety was more potent as it has prolonged the onset of convulsions by $18.7 \mathrm{~min}$. In particularly it has been found that if 4-substituted-aryl-8-substituted arylidene-2-imino-5,6-dihydro-4H,7H-(3,1)-benzothiazines with an increase in the number of hydrophobic electrons releasing or a combination of electron releasing and electron donating groups at different positions of the aryl and arylidene rings to increase the anticonvulsant activity further.

\section{ACKNOWLEDGEMENT}

The authors express their sincere gratitude to the management Vignan Institute of Pharmaceutical Sciences Hyderabad, for encouragement and for providing the essential requirements for carrying out this research work, and we also express our sincere thanks to Mr. Kadiri Sunil Kumar, Associate Professor, Department of Pharmacology, Vijaya College of Pharmacy, for help in pharmacological screening. We are extending our sincere and humble thanks to IICT Hyderabad, for helping in spectral analysis.

\section{CONFLICT OF INTERESTS}

The authors declare that they have no conflict of interest

\section{REFERENCES}

1. Thomas L Lemke, David A Williams. Foye's principles of medicinal chemistry. Philadelphia, P A Lippincott Williams and Wilkins; 2008.

2. Greene SA, Thurmon JC. Xylazine a review of its pharmacology and use in veterinary medicine. J Vet Phar Ther 1988;11:295-313.

3. Seeling A, Oelschlager H, Rothley D. Important pharmaceuticalchemical characteristics of the central muscle relaxant chlormezanone. Pharmazie 2000;55:293-6.

4. Ravindar B, Srinivasa Murthy M, Afzal Basha Shaik. Design, facile synthesis and biological evaluation of novel 1,3-thiazine derivatives as potential anticonvulsant agents. Asian J Pharm Clin Res 2016;9:272-6.

5. Ravindar Bairam, Srinivasa Murthy M. Synthesis characterization and biological screening of some novel substituted 1,3-thiazine derivatives. Der Pharm Chem 2015;7:150-4.
6. Shaik K Yazdan, Gali V Sagar, Afzal B Shaik. Biological and synthetic potentiality of chalcones. J Chem Pharm Res 2015; 7:829-42.

7. Rathore MM. Synthesis and antimicrobial activities of some bromo-substituted-1, 3-thiazines. Int J Res Pham Bio Sci 2013;4:59-62.

8. Silverstein R. Spectroscopic identification of organic compounds. $6^{\text {th }}$ ed. John Wiley and Sons Inc, New York; 1991.

9. Ibadur R Siddiqui, Pravin K Singh. Novel one pot synthesis of 1, 3-dithiins and 1, 3-thiazines under microwave irradiation. Int J Chem 2007;46:499-504.

10. Tarik E Sayed Ali, Azza Mohammed, E Kazak. Synthesis and antimicrobial activity of some new 1,3-thiazoles, 1,3,4thiadiazoles, 1,2,4-triazoles and 1,3-thiazines incorporating acridine and 1,2,3,4-tetrahydroacridine moieties. Eur J Chem 2010;1:23.

11. Srikanth Jupudi. Screening of in vitro anti-inflammatory activity of some newly synthesized 1,3-thiazine derivatives. Int J Res Pharm Chem 2013;3:213-20.

12. Vijay V Dabholkar, Sagar D Parab. 1, 3-Thiazines and 1, 3pyrimidines derivatives and their biological evaluation for antiinflammatory, analgesic and ulcerogenic activity. Hetero Lett 2011;1:176-88.

13. Udupi RH, Bhat AR, Jacob J. Synthesis and biological evaluation of some biphenyl ether and thiazine derivatives. Indian J Heterocycl Chem 2005;15:89.

14. Temple C, Wheeler GP, Comber RN, Elliot RD, Montgomery JA. Synthesis of potential anticancer agents, Pyrido[4,3b][1,4]oxazines and pyrido[4,3-b][1,4]thiazines. J Med Chem 1983;26:1641.

15. Subbagh-El, Abadi HI, Khawad IE, Pashood K. Synthesis of 2,2(1,4-phenylene)bis-3,4-dihydro-2H-1,3-thiazin-4-ones and their facile recyclization to 2,2-(1,4-phenylene)bis(pyrimidin4-one) and 2,2-(1,4-phenylene)-bis-(thieno[2,3-d] pyrimidin4(1H)-one) derivatives. Arch Pharm 1999;332:1148-54.

16. Roman Lesyk, Olena Vladzimirska, Serhiy Holota, Lucjusz Zprutko, Andrzej Gzella. New 5-substituted thiazolo[3,2b] $[1,2,4]$ triazol-6-ones. Synthesis and anticancer evaluations. Eur J Med Chem 2007;42:641-8.

17. Wei Wang, Bing Zhao, Chao Xu, Wenpeng Wu. Synthesis and antitumor activity of the thiazoline and thiazine multi thioether. Int J Org Chem 2012;2:117-20.

18. Beauchamp, Benardeau, Hilpert, Wang. 2-Aminodihydro 1,3thiazines as base 2-inhibitors. For the treatment of diabetes patent scope, World intellectual property organization; 2011. p. 165.

19. Zawisza. Syntheses and pharmacological analysis of new derivatives of tetra hydro-[1,3]-thiazine and 2-thiobarbituric acid. Nat-Cent Biot Inf 1981;29:235-48.

20. Nagihan B, Bedia KK, Salih G, Feyza A. Synthesis and anticonvulsant activity of some 2-pyrazolines derived from chalcones. Arabian J Chem 2013;44:1-9.

21. Keerthi Kumar B. Synthesis and biological evaluation of different thiazine derivatives. J Pharm Res 2011;4:274-5.

22. Vieira TM, Regitano-D'Arce MA. Ultraviolet spectrophotometric evaluation of corn oil oxidative stability during microwave heating and oven test. J Agric Food Chem 1999;47:2203-6. 
23. Foks H. Synthesis, structure and biological activity of 1, 2, 4triazolo-1,3-thiazine derivatives. Pharmaceutica 1992;47:770-3.

24. Bhusari PK, Khedekar PB, Umathe SN, Bahekar RH, Rama R. Synthesis of 8-bromo-9-substituted-1, 3-benzothiazolo-[5, 1b]-1, 3, 4-triazoles and their anthelmintic activity. Indian J Heterocycl Chem 2000;69:275.

25. Al-Khamees HA, Bayomi SM, Kandil HA, El-Thahir K. Microwave assisted green chemical synthesis of novel spiro [indole-pyrido thiazines], a system reluctant to be formed under thermal conditions. Eur J Med Chem 1990;25:103.

26. Turaska Rao, Bhongade SL, More SM, Dongarwar AS, Shende VS, Pande VB. Effects of lippia nodiflora extract on motor coordination, exploratory behaviour pattern, locomotor activity, anxiety and convulsions on albino Mice. Asian J Pharm Clin Res 2011;4:133-8.

27. Vogel AI. Text Book of Practical Organic Chemistry. $4^{\text {th }}$ ed. ELBS, London; 1986. p. 796.

28. Paxonikyan EG, Sirakanyan SN, Noravyan AS, Arzanunts EM, Paronikyan RG, Sarikisyan IS, et al. Synthesis of 8-benzylamino1, 2-dihydro-10-oxo-2, 2, 5-trimethyl-4H-pyrano [4, 3, 4, 5] pyrido [3, 2-e]-1, 3-thiazine and its psychotropic activity. Pharm Chem J 1994;28:25-8.

29. Dimmock JR, Kandepu NM, Hetherington M, Quail JW, Pugazhenthi U, Sudom AM. Cytotoxic activities of Mannich bases of chalcones and related compounds. J Med Chem 1998;41:1014-26.

30. Harode R, Sharma TC. Reaction of chalcone dibromide with thiourea in the presence of potassium hydroxide. Indian J Chem 1988;27B:1144-5.

31. Lipnick RL, Cotruvo JA, Hill RN, Bruce RD, Stitzed KA, Walker AP. Comparison of the up and down conventional LD50 and fixed dose acute toxicity procedures. Food Chem Toxicol 1955;33:223-31.

32. OECD Guidelines for the testing of chemicals, revised draft guideline 2001;423:1-14.

33. Parameshwar Ravula, Harinadha Babu V, Manichandrika P, Ramana Chary N, Narendra Sharath Chandra JN. Microwave assisted synthesis, biological evaluation and docking studies of novel pyrazoline derivatives as potent anti-inflammatory and antibacterial agents. Int J Chem Sci 2016;14:769-82.

34. Ahmed R Ali, Eman R El-Bendary, Mariam A Ghaly, Ihsan A Shehata. Novel acetamido thiazole derivatives, synthesis and in vitro anticancer evaluation. Eur J Med Chem 2013;69:908-18.

35. Holtkamp M, Meierkord H. Cellular and molecular pathogenesis of type 1A diabetes. Mol Life Sci 2007;64:2023-41.

36. Yamashita H, Ohno K, Amada Y, Hattori H, Funatsu YO, Toya T. Pharmacological and physiological functions of the polyspecific organic cation transporters: OCT1, 2, and 3 (SLC22A1-3). J Pharm Exp Ther 2004;308:127-33.

37. Bax BD, Chan PF, Eggleston DS, Fosberry A, Gentry DR, Gorrec $\mathrm{F}$, et al. Type IIA topoisomerase inhibition by a new class of antibacterial agents. Nature 2010;466:935-40.

38. Richard A, Friesner Jay L, Banks, Robert B Murphy, Thomas A Halgren, Jasna J Klicic, et al. Glide: a new approach for rapid, accurate docking and scoring. 1. Method and assessment of docking accuracy. J Med Chem 2004;47:1739-49.

39. Dongamanti Ashok, Kavitha Rangu, Velagapuri Hanumantha Rao, Srinivas Gundu, Ballu Srilata, Manga Vijjulatha. Medicinal Chemistry Research; 2016;25:501-14.

40. Hargrove TY, Kim K, De Nazaré Correia Soeiro M, Da Silva CF, Batista DD, Batista MM, et al. CYP51 structures and structure-based development of novel, pathogen-specific inhibitory scaffolds. Int J Parasitol: Drugs Drug Resist 2012; 2:178-86.

41. Rayko Becher, Stefan GR Wirsel. Fungal cytochrome P450 sterol 14 alpha-demethylase [CYP51] and azole resistance in plant and human pathogens. Appl Micro Biot 2012;95:825-40.

42. White P, Aberle H, Vincent JP. Signaling and adhesion activities of mammalian beta-catenin and plakoglobin in drosophila. Cell Biol 1998;140:183-95.

\section{How to cite this article}

- Ravindar Bairam, Srinivasa Murthy Muppavarapu, Sivan Sreekanth. Synthesis, characterization, biological evaluation and docking of some novel substituted 1, 3-thiazine derivatives. Int J Pharm Pharm Sci 2017;9(3):233-242. 